\title{
The effects of global grouping laws on surface lightness perception
}

\author{
SONGJOO OH and JUNG-OH KIM \\ Seoul National University, Seoul, South Korea
}

\begin{abstract}
Previous studies of lightness perception have shown that local surface grouping laws such as proximity and T junction were powerful determinants of target surface lightness. Recent lightness theories also emphasize the importance of local grouping of surfaces. In this study, we further examined the effects of three global grouping laws-symmetry, repetition, and alternation-on lightness perception. Local surface grouping laws such as proximity and good continuation were controlled across all of our stimulus displays. Participants' lightness perception consistently depended on a given surface's belongingness as determined by these laws-that is, global grouping laws affected a target surface's lightness perception. Our results indicate that global grouping laws determine a target surface's lightness when local surface grouping does not produce any distinct surface belongingness. Implications of our basic results are discussed in terms of a recent lightness theory.
\end{abstract}

How do we perceive the lightness of a surface in an environment bombarded by varying illuminations? How is retinal luminance processed into a black-white-luminous dimension? There have been three prominent approaches to this time-honored issue: lateral inhibition, ratio rule, and intrinsic image analysis. The lateral inhibition theory proposed that surface lightness is achieved by an inhibitory interaction of neighboring retinal receptors. Although this theory has advantages such as quantifiable predictions, it cannot provide neat explanations for lightness perception in complex scenes. The ratio rule hypothesis (Wallach, 1948), inspired by gestalt grouping laws, emphasized the luminance ratio between the target and its surround in lightness constancy. Although ample neurophysiological and computational evidence supports this hypothesis, it cannot adequately explain lightness perception in a complex 3-D layout (see, e.g., Gilchrist, 1977). Intrinsic image analysis, first proposed by Helmholtz (1868/1962), assumes the importance of factoring illuminations from surface reflectance. All these theories have problems explaining diverse lightness perception phenomena (for a recent critical review, see Gilchrist et al., 1999).

Gilchrist et al. (1999) proposed a comprehensive theory of surface lightness perception. Their anchoring theory emphasizes the importance of solving two problems in surface lightness: anchoring and scaling. An anchoring problem arises when specific values of lightness are

The authors gratefully acknowledge Alan Gilchrist's valuable comments on earlier drafts of the manuscript. S.O. is now at Rutgers University. Correspondence concerning this article should be addressed to J.-O. Kim, Department of Psychology, College of Social Sciences, Seoul National University, Seoul 151-742, South Korea (e-mail: jungokim @plaza.snu.ac.kr).

Note-This article was accepted by the previous editorial team, headed by Neil Macmillan. assigned to surfaces; a scaling problem (which is minor) arises when these values are distributed. Because the ratio of the luminance of a target surface to that of its surround is relative, our perception of the target's lightness cannot be based on this ratio. We thus need anchoring rules such as "high luminance = whiteness." This rule prescribes that among surfaces of varying luminances, the surface with the highest luminance is perceived as white, even when it is actually gray. When the lightness of a given surface in complex images must be determined, both local and global anchoring occurs in a framework consisting of a group of surfaces. The belongingness of a surface to a given framework follows gestalt laws of grouping such as proximity, good continuation, and similarity. By taking into account the ratio of the target to the highest luminance in both the local and the global frameworks, the anchoring theory was very successful in explaining, quantitatively as well as qualitatively, a wide range of lightness perception phenomena.

In simultaneous lightness contrast, a gray square surrounded by a black background is perceived as lighter than a square of the same shade of gray surrounded by a white background. The anchoring theory explains this phenomenon as follows: Global anchoring sets these two squares at a given level of surface gray, but local anchoring sets the former gray square as much whiter than the latter. A compromise between the two anchoring results produces a typical simultaneous lightness contrast. The same theory also explains the Benary illusion as follows: A gray triangle within an arm of a black cross looks lighter than an identical gray triangle abutting the two arms of the same cross. Because the interior gray triangle belongs to the black cross and the exterior gray triangle belongs to the white background, the former triangle is perceived as being a much lighter shade of gray than the latter. To be specific, the anchoring theory pro- 
poses that a T-junction analysis of surfaces into those that occlude and those that are occluded should be responsible for surface grouping. That is, lightness contrast takes place between the two occluded surfaces. The interior gray surface contrasts sharply with part of the black cross, whereas the exterior gray surface contrasts with part of the white background.

Unlike previous lightness perception theories such as lateral inhibition and the ratio rule, the anchoring theory emphasizes geometric factors - that is, surface belongingness on calculations of surface lightness. Gestalt factors such as proximity, good continuation, and $\mathrm{T}$ junction contribute to grouping of surfaces in complex images. These factors are local in nature. For instance, a T-junction analysis focuses on horizontal, vertical, and slanting features without considering global factors such as symmetry (see Ross \& Pessoa, 2000, for a recent in-depth look at this analysis).

Leeuwenberg and Boselie (1988) proposed that some grouping factors, such as regularity, symmetry, and alternation, are formal aspects similar to the minimum principles in form organization, whereas others, such as proximity, depth cues, and area, are their contents. Lightness and its constancy are also contents. Such a categorization of gestalt grouping laws indicates that grouping of surfaces in a local framework leaves much room for grouping of surfaces in the global framework. One important question is raised at this point: Are the global grouping laws also responsible for surface lightness perception? The anchoring theory of lightness perception has dealt only with global frameworks such as surrounds of varying luminances and white backgrounds (e.g., a page of a book and the table on which the book is lying, respectively). We propose that gestalt laws such as regularity, repetition, and symmetry, being located between the local and global frameworks as proposed in the anchoring theory, should dynamically contribute to the anchoring processes by facilitating surface grouping. If these laws contribute to surface lightness perception independently of local grouping laws (e.g., T junction, proximity), the applicability of the anchoring theory can be greatly extended to various surface lightness situations. What if two local T-junction analyses produced the same grouping of surfaces? In this case, surface grouping should occur at the global level. In our study, local factors are defined as gestalt laws such as proximity and $\mathrm{T}$ junction, and global factors are defined as gestalt laws such as symmetry, repetition, and alternation. Using visual displays in which local factors are controlled, we explored the effects of following global factors on the perception of surface lightness (Experiments 1-3), symmetry (Experiment 1), repetition (Experiment 2), and alternation (Experiment 3 ). The results of our experiments should further uncover the nature of surface grouping, which has been regarded as crucial by the anchoring theory (Gilchrist et al., 1999) as well as by a selective integration model (Ross \& Pessoa, 2000).

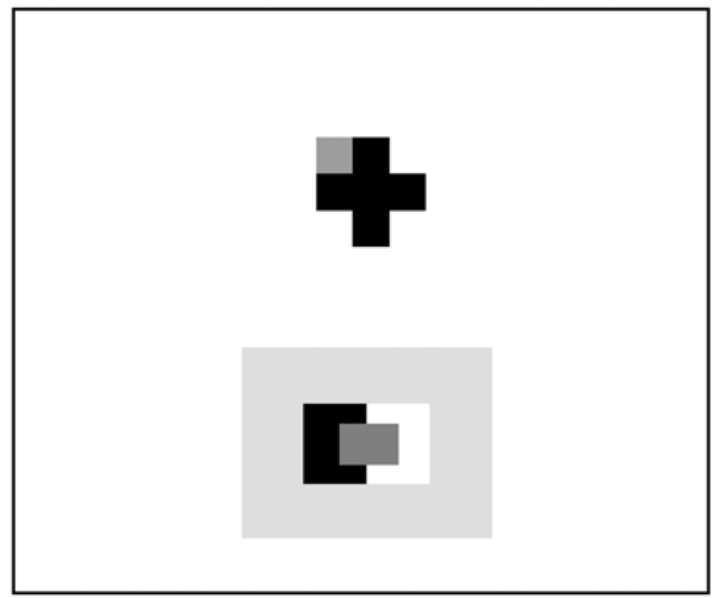

Figure 1. Test display (top) and matching box (bottom) presented across all of the experiments.

\section{GENERAL METHOD}

\section{Participants}

Students at Seoul National University participated in each experiment as a partial requirement of courses in introductory psychology and human relations. They had not participated in any previous lightness experiment.

\section{Procedure}

All the experiments were conducted in a dark room. Each participant was dark adapted for about $3 \mathrm{~min}$, after which he or she was told about the experimental procedures and instructed to respond with a pair of keys. All the stimuli were displayed on a high-resolution, 21-in. monitor screen (Mitsubishi Diamond Pro21 tx) under the control of a Sambo Dreamsys $7500 \mathrm{H}$ Pentium Computer. A black target $\left(1.45 \mathrm{~cd} / \mathrm{m}^{2}\right.$ in luminance $)$ and a fixed midgray target $\left(26.9 \mathrm{~cd} / \mathrm{m}^{2}\right.$ in luminance) were displayed on a white background $\left(66 \mathrm{~cd} / \mathrm{m}^{2}\right)$. The participants practiced once with a simple gray rectangle $(6 \times$ $5 \mathrm{~cm}$ ). Each experiment started with a test screen (see Figure 1). A test display occupied the upper half of the screen, and a matching box occupied the lower half.

Each participant's lightness responses were collected by means of an adjustment method. He or she was to make the lightness of the matching box equal to that of the test display. The luminance of the matching gray patch started randomly at one of the following nine levels: $16.4,18.9,21.6,23.8,26.9,33.4,36.0,39.6$, and $42.8 \mathrm{~cd} / \mathrm{m}^{2}$. The luminance of the test and that of the matching display were measured by a Chroma meter (Minolta cs 100). Three responses were collected from each participant for each of these displays. After finishing the first session, each participant took a 2-min rest under normal fluorescent lightning. He or she was then dark adapted for 2-3 min and completed the second session.

In each experiment, each participant judged two test displays. For half of the participants, one test display was followed by another, and for the remaining half the order was reversed. The participants were instructed to view an upper display and to match a gray in the lower display to the test gray. The distance between the monitor and each participant was about $70 \mathrm{~cm}$. The displays were viewed binocularly.

\section{Instruments and Displays}

The actual display screen was $36 \times 31 \mathrm{~cm}$ in size, and the left part of the monitor screen was hidden from the participant by a rec- 
A

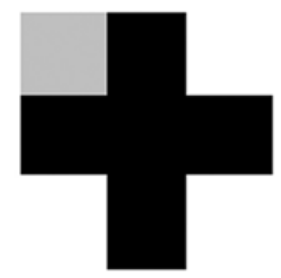

a

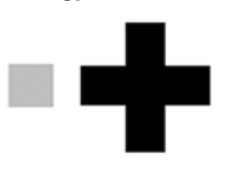

$a^{\prime}$

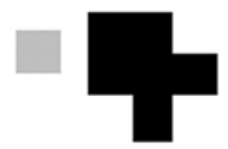

B

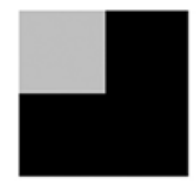

b

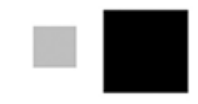

$b^{\prime}$

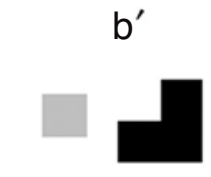

Figure 2. Cross (A, a, and $\left.a^{\prime}\right)$ and square $\left(B, b\right.$, and $\left.b^{\prime}\right)$ displays presented in Experiment 1.

tangular paper, under which the control display was placed. The experimenter controlled the entire experiment using the control display and a mouse.

The test and matching displays were presented simultaneously when the experimenter clicked on the mouse. If the participant pressed the left key, the gray patch in the matching display slowly became darker. If he or she pressed the right key, the same surface slowly became lighter. Each keypress led to about $0.25-0.3 \mathrm{~cd} / \mathrm{m}^{2}$ in luminance change. The matching display was placed at the center of a black and white surface and mounted on a light gray target $\left(54.8 \mathrm{~cd} / \mathrm{m}^{2}\right)$. This display was similar to the one used by Schirillo, Reeves, and Arend (1990).

\section{EXPERIMENT 1 \\ The Effects of Symmetry on Surface Lightness Perception}

A minimum principle emphasizes the importance of symmetry in the perception of forms, partly because a symmetrical pattern results in the simplest and most economical description regarding its structure (see, e.g., Helm \& Leeuwenberg, 1996; Leeuwenberg \& Boselie, 1988). In
Experiment 1, we explored whether symmetry influences lightness perception when local grouping factors such as $\mathrm{T}$ junction and good continuation are controlled.

The cross and the square in Figure 2 were identical in both the $\mathrm{T}$ junctions and good continuation conditions. Each figure has two $\mathrm{T}$ junctions, one at the left and the other in the top position. Grouping by symmetry should allow perceptual preference for $a$ in the interpretation of a black cross and for $b^{\prime}$ in the interpretation of a black square. The gray surface in the cross should be perceived as belonging to a white background, whereas the same surface in the square should be perceived as belonging to the black square. Such surface grouping by symmetry should result in the perception of a lighter gray in the square than in the cross.

To further explore surface grouping by symmetry, we designed a new set of figures, shown in Figure 3. Following the logic above, the gray in the square should appear lighter than the gray in the octagon. If good continuation leads to surface grouping, it will be difficult to predict which gray looks lighter.

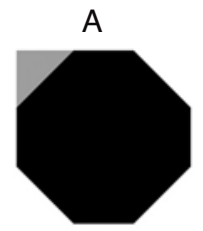

a
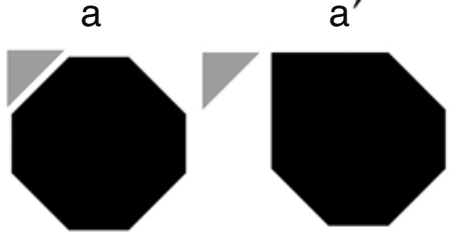
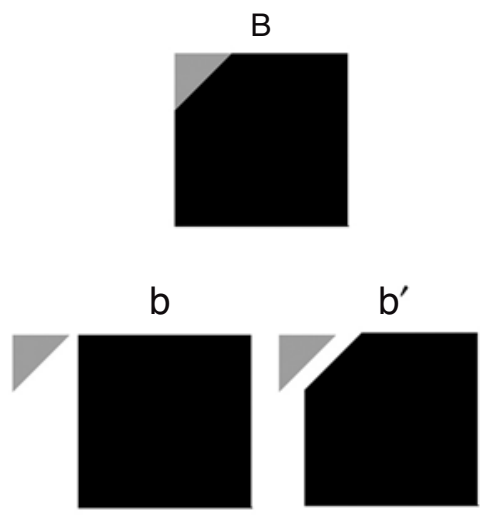

Figure 3. Octagon (A, a, and $\left.a^{\prime}\right)$ and the square $\left(B, b\right.$, and $\left.b^{\prime}\right)$ displays presented in Experiment 1. 
Table 1

Mean Luminance (and Standard Deviation) to Each Test Gray by Display: Experiment 1 (Symmetry)

\begin{tabular}{clcc}
\hline Figure & Display & $M\left(\mathrm{~cd} / \mathrm{m}^{2}\right)$ & $S D$ \\
\hline 2 & Cross & 25.3 & 2.0 \\
\multirow{2}{*}{3} & Square & 26.8 & 1.1 \\
& Octagon & 25.5 & 3.1 \\
& Square & 26.4 & 3.2 \\
\hline
\end{tabular}

\section{Method}

Participants. Twelve undergraduate students at Seoul National University responded to the displays in Figure 2, and another group of 12 students responded to those in Figure 3. All of them had vision that was normal or corrected to normal. It took each of them about 15 min to finish each session.

Displays. The black cross (Figure 2) was $4.7 \times 4.7 \mathrm{~cm}\left(3.85^{\circ}\right)$, the small gray square was $3.1 \times 3.1 \mathrm{~cm}\left(2.54^{\circ}\right)$, the large black square was $4.7 \times 4.7 \mathrm{~cm}$, and the octagon $4.7 \times 4.7 \mathrm{~cm}$ in size. The test gray square was $1.5 \times 1.5 \mathrm{~cm}$, and the side of the test gray triangle was also $1.5 \mathrm{~cm}$ in length.

\section{Results and Discussion}

The mean luminance that the participants produced in response to each test display is presented in Table 1 . The mean lightness of the test gray in the square of Figure 2 was significantly higher than the mean lightness of the test gray in the cross $[t(11)=2.69, p=.025]$. The mean lightness of the test gray in the square of Figure 3 was $0.9 \mathrm{~cd} / \mathrm{m}^{2}$ higher than the mean lightness of the test gray in the octagon. This difference was, however, not significant $[t(11)=$ $1.87, p=.08]$. Several participants reported that they were confused when viewing Figure $3 \mathrm{~A}$ - that is, they were not sure whether they were seeing a very short pencil or an octagon. Such an interpretation of this display may have interfered with grouping by similarity for the octagon.

The results of Experiment 1 clearly show that surface grouping by symmetry significantly affects lightness perception. That is, depending on whether or not the gray of the target is perceived as belonging to a black surface, its lightness perception varies, even though a local $\mathrm{T}$ junction and good continuation were identical across two test displays. In particular, a coplanarity rule, here defined by a Tjunction analysis, cannot predict different lightness perceptions between displays.

Are there any other possible explanations for the results of Experiment 1? Inspection of Figures 2 and 3 may suggest the importance of the factor of area in determining surface lightness. That is, the gray surface neighboring a large black area should look lighter than the gray surface neighboring a small black area. Following this hypothesis, the target in the cross should be lighter than that in the square in Figure 2, and the target in the square should be lighter than that in the octagon in Figure 3. The pattern of results is, however, not consistent with this alternative explanation.

\section{EXPERIMENT 2 \\ The Effects of Repetition on Surface Lightness Perception}

Leeuwenberg and Boselie (1988) included repetition as one important formal aspect of gestalt grouping. In Experiment 2, we explored whether repetition of surfaces influences lightness perception when the T-junction factor is controlled. We first examined whether repetition of the same form determines the belongingness of the gray surface, and then explored whether context repetition also determines surface grouping in lightness perception.

The two displays shown in Figures 4A and 4B are interpreted by the repetition law as two adjacent parallelograms, although their surface lightness differed in opposite ways. The gray triangle in panel $\mathrm{A}$ is perceived as belonging to the white parallelogram, and the identical triangle in panel $\mathrm{B}$ is perceived as belonging to the black parallelogram. Although the triangles have the same $\mathrm{T}$ junctions, they differed only in the arrangement of lightness surfaces. Following the minimum principle of preference for a small number of parameters, panels A and B should be interpreted as $a$ and $b$ (middle) as op-

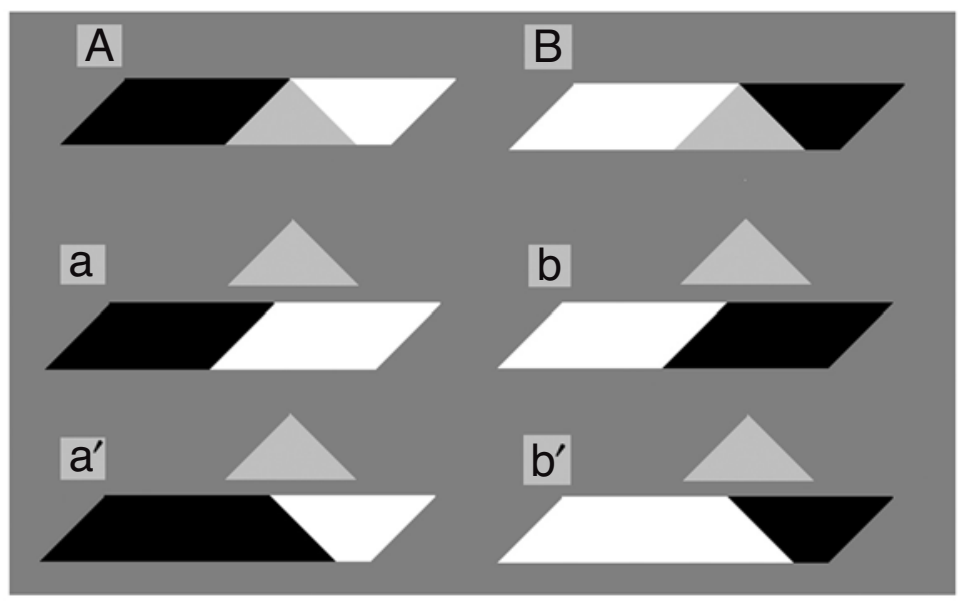

Figure 4. Parallelogram displays presented in Experiment 2. 
A
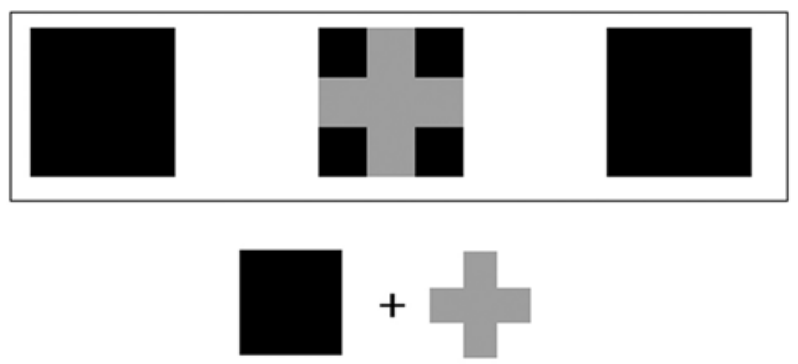

B
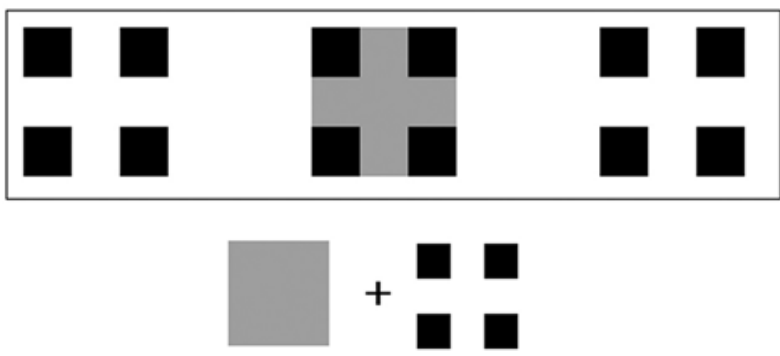

Figure 5. Cross-square displays presented in Experiment 2.

posed to $a^{\prime}$ and $b^{\prime}$ (bottom), in which surfaces do not repeat. According to the latter interpretations, nonrepetition of surfaces prevails. If this were the case, the gray triangle in panel B should look lighter than the same triangle in panel $\mathrm{A}$.

Repetition of a context, like repetition of the target figure, should work in the same way in surface grouping. The central figure in Figures 5A and 5B has two $\mathrm{T}$ junctions at each of the left, top, bottom, and right positions. The perceptual interpretations, however, are different in terms of the repetition of different contexts: A gray cross is perceived as being on a large black square in panel $\mathrm{A}$, whereas four small squares are perceived as placed on a large gray square in panel B. If these perceptual interpretations are correct, then the gray cross in panel A should look lighter than the same gray cross in panel B.

\section{Method}

Participants. Twelve undergraduate students at Seoul National University participated in the parallelogram display condition, and another group of the same number participated in the cross-square display condition.

Procedure. The procedure described in the General Method was used in Experiment 2.

Instruments and displays. The test and the matching displays for the parallelogram condition were presented on a gray background $\left(15.7 \mathrm{~cd} / \mathrm{m}^{2}\right)$. Each parallelogram was $4 \mathrm{~cm}\left(3.28^{\circ}\right)$ at the base and $1.6 \mathrm{~cm}\left(1.31^{\circ}\right)$ high. The gray triangle was $3 \mathrm{~cm}\left(2.46^{\circ}\right)$ at the base and $1.6 \mathrm{~cm}\left(1.31^{\circ}\right)$ high. The test and the matching displays for the cross-square condition were identical to those in Experiment 1 . The black squares in Figure 5 were $3 \times 3 \mathrm{~cm}$, and the small squares were $1 \times 1 \mathrm{~cm}\left(0.82^{\circ}\right)$ in size.

\section{Results and Discussion}

The mean luminance that the participants produced in response to each test display is presented in Table 2. In the parallelogram condition, the mean lightness for the triangle in Figure 4B was significantly higher than the mean lightness for that in Figure 4A $[t(11)=2.33, p=$ $.04]$. The participants in the cross-square condition perceived the cross in Figure 5A as lighter than that in Figure $5 \mathrm{~B}[t(11)=3.39, p=.005]$. The results from these two sets of figures indicate that repetition of the target figure and that of the context influence surface lightness when a local T-junction analysis produces identical surface grouping. Like surface grouping by symmetry, surface grouping by repetition also influences lightness perception when a local analysis cannot resolve in favor of a unique belongingness of the target surface.

In comparison with the effects of surface grouping by symmetry, those of grouping by repetition appear very effective in that the pattern of results in Figures 4 and 5 was consistent with the prediction based on the global grouping laws. This result should be due to a regular repetition of identical surfaces.

\section{EXPERIMENT 3 \\ The Effects of Alternation on Surface Lightness Perception}

Agostini and Proffitt (1993) reported different lightness perceptions of the gray circles when each target was embedded in alternating columns of black and white circles. That is, a gray circle belonging to a column of white circles looks darker than an identical gray circle belonging to a column of black circles. This result suggests that grouping by good continuation affects target surface lightness. The same explanation applies to the checkerboard lightness contrast reported by De Valois and De Valois (1988). In this contrast, a gray square surrounded by black squares appears darker, whereas the same gray square surrounded by white squares appears lighter. A diagonal grouping of the gray with the white squares and another grouping of the gray with the black squares should be responsible for this lightness contrast.

What Agostini and Proffitt (1993) did not consider is an analysis of a horizontal alternation of black and white circles and their effects on target surface lightness. That is, belongingness in a column grouping was emphasized in Agostini and Proffitt's study. The same argument can

Table 2

Mean Luminance (and Standard Deviation) of Each Test Gray by Display: Experiment 2 (Repetition)

\begin{tabular}{cccc}
\hline Figure & Display & $M\left(\mathrm{~cd} / \mathrm{m}^{2}\right)$ & $S D$ \\
\hline \multirow{2}{*}{4} & Parallelogram A & 31.3 & 1.6 \\
& Parallelogram B & 32.6 & 2.4 \\
5 & Cross-square A & 23.8 & 1.9 \\
& Cross-square B & 22.6 & 1.8 \\
\hline
\end{tabular}




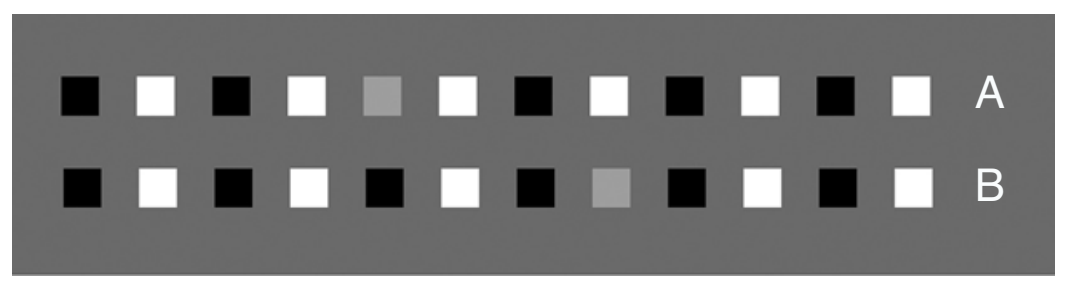

Figure 6. Small squares displays presented in Experiment 3.

be used in explaining De Valois and De Valois's (1988) lightness contrast. In Experiment 3, we explored whether a horizontal alternation of surfaces of different degrees of lightness influences target surface lightness. In Figure 6, the upper series of small squares (panel A) and the lower series of small squares (panel B) differ in grouping of white, black, and gray surfaces: If surface grouping by alternation works, the gray square in panel A should belong to the black row, whereas the gray square in panel $\mathrm{B}$ should belong to the white row. The gray square in panel A, belonging to the black row, should be perceived as much lighter than that in panel B, belonging to the white row.

To further examine the generality of surface grouping by alternation, we examined lightness differences in bull'seye $^{1}$ displays (Figure 7). The central gray disk in the left bull's-eye display (panel A) should be grouped by alternation with the white ring surfaces, whereas an identical central gray disk in the right bull's-eye display (panel B) should belong with the black ring surfaces. The central gray disk in the right bull's-eye display should thus appear lighter than should the central gray disk in the left bull'seye display.

\section{Method}

In Experiment 3, we employed two measurement methods: an adjustment and a simultaneous comparison. Twenty-eight participants used the adjustment method in responding to the test display. Half of them received the display in Figure 6, and the remaining half received the display in Figure 7.

Each of the 12 squares in each of the two displays in Figure 6 was $1 \mathrm{~cm} \times 1 \mathrm{~cm}\left(0.82^{\circ}\right)$ in size, and they were separated by a $1-$ $\mathrm{cm}$ interval. The luminance for the white surfaces in a dozen of the

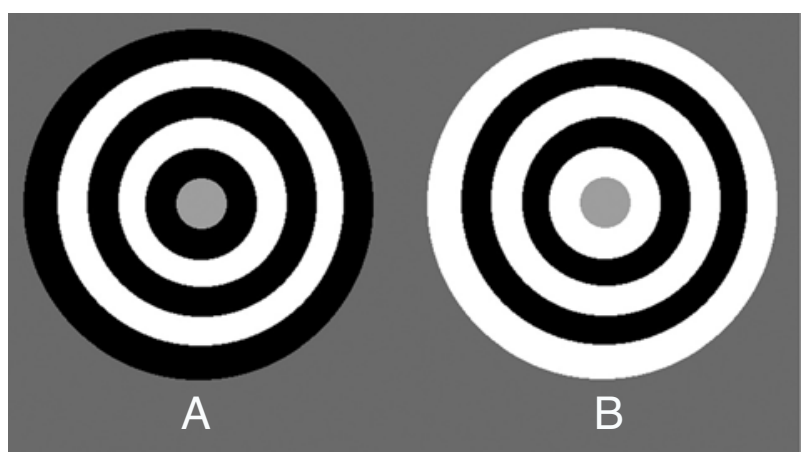

Figure 7. Bull's-eye displays presented in Experiment 3. squares in Figure 6 was $26.9 \mathrm{~cd} / \mathrm{m}^{2}$, and the luminance for the background was $15.7 \mathrm{~cd} / \mathrm{m}^{2}$. In Figure 7, the luminance for the central gray disk, which was $1.5 \mathrm{~cm}\left(1.23^{\circ}\right)$ in diameter, was $26.9 \mathrm{~cd} / \mathrm{m}^{2}$, and the luminance of its background was $15.7 \mathrm{~cd} / \mathrm{m}^{2}$. Each of the white and black rings of the bull's-eye displays was $1 \mathrm{~cm}\left(0.82^{\circ}\right)$ in width.

Partly because the participants using the adjustment method tended to focus on the gray of the target rather than notice a regular alternation of surfaces, a new group of 24 participants was enlisted to respond to the test displays in Figures 6 and 7 by the use of simultaneous comparison. These participants were instructed to pick up the test display that looked lighter. The two test displays shown in Figure 6 were presented one above the other with a distance of $1.7 \mathrm{~cm}$ between them. The position of each display was counterbalanced across participants. The two test displays shown in Figure 7 were presented horizontally, with their left and right positions counterbalanced across participants.

\section{Results and Discussion}

The mean luminance that the participants produced in response to each test display is presented in Table 3. No difference in lightness judgment was seen for the square series displays $[t(13)=.33, p=.54]$. The participants who were given the bull's-eye displays perceived Figure $7 \mathrm{~B}$ as much lighter than Figure $7 \mathrm{~A}[t(13)=3.83$, $p=.002]$. The results for the simultaneous comparison are presented in Table 4 . Nineteen of the 24 participants who were given the square series displays picked Figure 7A over Figure 7B $\left[\chi^{2}(1)=8.17, p=.004\right]$. Twenty of the 24 participants who were given the bull's-eye displays preferred panel $\mathrm{B}$ over panel $\mathrm{A}\left[\chi^{2}(1)=10.6, p=\right.$ $.001]$.

The divergence of the results in the lightness judgment in Experiment 3 are due to the participants' mode of attention. That is, the adjustment method might encourage participants to focus locally on the target square, thus minimizing the effectiveness of grouping by surface alternation. Using a different task, Tsal, Shalev, Zakay, and Lubow (1994) showed that attention reduces perceived brightness contrast. The simultaneous comparison method should have prevented this from happening when the participants examined the test displays.

The pattern of results of Experiment 3 might, however, arise from lightness assimilation (or a spreading effect) induced by adjacent squares or rings. The visual angle of the gray target patches and that of the inducing patches in Experiment 3 were almost identical. Classic lightness assimilation effects such as von Bezold spreading are usually produced in gray regions containing tiny black or white textural elements. ${ }^{2}$ Such displays are not like those 
Table 3

Mean Luminance (and Standard Deviation) of Each Test Gray by Display: Experiment 3 (Alternation)

\begin{tabular}{clcc}
\hline Figure & Display & $M\left(\mathrm{~cd} / \mathrm{m}^{2}\right)$ & $S D$ \\
\hline \multirow{2}{*}{7} & Square A & 33.8 & 3.0 \\
& Square B & 33.4 & 3.6 \\
& Bull's-eye A & 26.6 & 3.5 \\
& Bull's-eye B & 31.7 & 3.5 \\
\hline
\end{tabular}

we used in Experiment 3, in which the target and the inducing regions are on the same scale. When the gray target was displayed only with two adjacent patches, we were able to observe a powerful simultaneous contrast.

Recently, Bindman and Chubb (in press) demonstrated a very powerful assimilation effect with square-form bull's-eye displays, which are quite similar to ours (Figure 7). Interestingly, their assimilation effect was affected neither by the width of the black and white surround nor by the contrast of a reference target. This effect was, however, systematically influenced by the number of surround bands. Although Bindman and Chubb rejected the anchoring hypothesis, we argue that a global surface grouping by alternation might operate on Bindman and Chubb's bull's-eye displays. This conclusion seems quite reasonable because they found that target lightness was systematically changed by the number of surround bands. Many participants in our experiment reported that the black and white squares of Figure 6 as well as the black and white rings of Figure 7 were easily seen as two groups, as if the lightness patches were arranged at different depths. Further research is thus warranted to examine whether Bindman and Chubb's effect arises from global surface grouping by alternation.

The pattern of results in Experiment 3 suggests that surface grouping by local proximity can easily be overridden by surface grouping by alternation at a global level. The results of Experiment 3 are consistent with those of Experiments 1 and 2 in showing the importance of global surface grouping in determining the belongingness of the target surface in the framework as a whole.

\section{GENERAL DISCUSSION}

The three experiments of the present study showed that global surface grouping laws such as symmetry, repetition, and alternation influenced target surface lightness when the T-junction analysis and other local grouping factors were controlled. Because surfaces surrounding the target gray were almost identical across these exper-

Table 4

Numbers and Percentages of Participants Choosing Each Display as Lighter: Experiment 3 (Alternation)

\begin{tabular}{clcc}
\hline Figure & Display & No. Participants & Percentage \\
\hline \multirow{2}{*}{6} & Square A & 19 & 80 \\
& Square B & 5 & 20 \\
7 & Bull's-eye A & 4 & 17 \\
& Bull's-eye B & 20 & 83 \\
\hline
\end{tabular}

iments, our findings cannot be explained by local cell activity. Because local surface grouping factors (e.g., T-junction analysis, good continuation) were also identical in the two figures in each set of test displays, they should not be responsible for our findings. This conclusion, however, does not imply that local surface grouping factors are not important. Except in Experiment 3, lightness differences between the two figures in each set were about $1-2 \mathrm{~cd} / \mathrm{m}^{2}$, which are smaller than those reported in previous work on lightness in which $\mathrm{T}$ junctions, coplanarity, and other local factors were varied. The small treatment effects should thus arise from the operation of global surface grouping laws, independently of local surface grouping laws.

Studies emphasizing local grouping factors usually contain two gray targets within an object (e.g., a cross or a set of black and white bars). Depending on the organization of parts of a given form, two gray targets produce different surface groupings. In contrast, our test displays consist of separate objects or a set of surfaces. What they have in common is that they both produce the same surface grouping when the T-junction analysis is applied. Global surface grouping laws should thus operate in the computation of target surface lightness. In this context, the results of our study uncover a possible missing link between local surface grouping and the global framework.

Global surface grouping by alternation warrants further discussion. Unlike other global laws such as symmetry and repetition, this law concerns multiple texture-like surfaces rather than object-like surfaces. By counteracting proximity and good continuation, grouping by alternation seems a bit more abstract in comparison with other global laws. The fact that this law was confirmed in the simultaneous comparison method also suggests its globality in lightness computation. Future work is warranted to discriminate between global surface grouping laws.

The pattern of lightness results across the three experiments shows that among three global grouping laws, repetition and alternation are more effective than symmetry in contributing to surface lightness. Repetition and alternation, common in the repetition of identical surfaces, are qualitatively different from symmetry, which is applied to a single large surface in Experiment 1. Symmetry, in combination with repetition, could significantly contribute to surface grouping. The relative contributions of these grouping laws should be explored in future work.

Our results in three experiments indicate that the categorization of gestalt laws into global versus local laws is quite productive as well as valid in understanding surface lightness perception. This categorization is valid in the sense that global grouping laws have their own psychological reality in contributing to the lightness of a target surface. It is also productive in the sense that it leads to new findings with respect to lightness, independently of local grouping laws (see Agostini \& Galmonte, 2002, for a different approach to perceptual belongingness).

Because our new finding indicates an independent operation of global surface grouping laws, the anchoring processes should become flexible but complicated in the 
computation of the target surface. This is partly because the anchoring theory (Gilchrist et al., 1999) has been successfully applied to such surface lightness displays as twofield domes, disk-ring displays, and Mondrian patches. Our lightness displays, however, allowed gestalt global grouping laws to operate on them, whereas local laws did not produce any distinct surface groupings. It is likely that global grouping laws could affect local surface lightness. Further empirical work is needed to explore the dynamic relationships among three levels of surface groupinglocal grouping, global grouping, and global frameworkrather than between the first two only.

Are global grouping laws obligatory or optional in affecting lightness judgment? Given a random organization of surfaces, as in Mondrian patches, they should be optional in that both local and global frameworks should be anchored. On the other hand, a visible environment often consists of regular, repeated surfaces such as rectangular walls and textured floors. Global grouping laws should suggest solutions for target surface lightness. This should be the case especially when local surface groupings might not work, simply because surfaces are large and repetitive. Global surface grouping laws may be more ecologically valid than local grouping laws. This and other issues warrant further examination.

\section{REFERENCE}

Agostini, T., \& Galmonte, A. (2002). Perceptual organization overcomes the effects of local surround in determining simultaneous lightness contrast. Psychological Science, 13, 89-93.

Agostini, T., \& Proffitt, D. R. (1993). Perceptual organization evokes simultaneous lightness contrast. Perception, 22, 263-272.
Bindman, D., \& ChubB, C. (in press). Brightness assimilation in bullseye displays. Vision Research.

De Valois, R. L., \& De Valois, K. K. (1988). Spatial vision. New York: Oxford University Press.

GILCHRIST, A. (1977). Perceived lightness depends on perceived spatial arrangement. Science, 195, 185-187.

Gilchrist, A., Kossfidis, C., Bonato, F., Agostini, T., Cataliotti, J., Li, X., Spehar, B., Annan, V., \& Economou, E. (1999). An anchoring theory of lightness perception. Psychological Review, 106, 795-834.

Helm, P., \& Leeuwenberg, E. (1996). Goodness of visual regularities: A nontransformational approach. Psychological Review, 103, 429456.

Helmholtz, H. von (1962). The recent progress in the theory of vision. In M. Kline (Ed.), Popular scientific lectures (pp. 93-185). New York: Dover. (Original work published 1868)

Leeuwenberg, E., \& Boselie, F. (1988). Against the likelihood principle in visual form perception. Psychological Review, 95, 485-491.

Ross, W. D., \& PessoA, L. (2000). Lightness from contrast: A selective integration model. Perception \& Psychophysics, 62, 1160-1181.

Schirillo, J., Reeves, A., \& Arend, L. (1990). Perceived lightness, but not brightness, of achromatic surfaces depends on perceived depth information. Perception \& Psychophysics, 48, 82-90.

Tsal, Y., Shalev, L., ZaKaY, D., \& Lubow, R. (1994). Attention reduces perceived brightness contrast. Quarterly Journal of Experimental Psychology, 47A, 865-893.

WALLACH, H. (1948). Brightness constancy and the nature of achromatic colors. Journal of Experimental Psychology, 38, 310-324.

\section{NOTES}

1. The authors are grateful to Alan Gilchrist for pointing out that our Figure 7 was very similar to Bindman and Chubb's (in press) bull's-eye patterns, and to Daniel Bindman and Charles Chubb for sending us their manuscript.

2. This point was raised by Alan Gilchrist.

(Manuscript received November 4, 2002; revision accepted for publication October 11,2003.) 